Abstract 141 Table 3 Expert-identified potential increased risk from device highlighting ( $\max n=103$ )

\begin{tabular}{|l|c|c|}
\hline & Yes & No \\
\hline Nerve Injery (103) & $5(4.5 \%)$ & $\$ 8(95.1 \%)$ \\
\hline Pacemocherax (65) & $0(0 \%)$ & $65(100 \%)$ \\
\hline LAST (86) & $3(3.5 \%)$ & $\$ 3(96.5 \%)$ \\
\hline Peritoncum Violation (149) & $0(0 \%)$ & $49(100 \%)$ \\
\hline Bluck Failere (103) & $\mathbf{4 ( 3 . 5 \% )}$ & $\$ 9(96.1 \%)$ \\
\hline Tetal (406) & $\mathbf{1 2 ( 3 . 0 \% )}$ & $\mathbf{3 9 4}(\mathbf{9 7 . 0 \% )}$ \\
\hline
\end{tabular}

$=0.06)$. Negative feedback was noted in 9/360 (2.5\%) and $20 / 300(6.7 \%)$ for non-experts and experts (Yates's $\chi^{2}=$ $5.81, \mathrm{p}=0.02)$. Experts reported no increased risk in 394/ $406(97.05 \%)$ and that unnoticed incorrect device highlighting carried potential increased risk in 12/406 (3.0\%).

Conclusions Non-experts viewed the device most positively, particularly in support of learning, training and identifying sono-anatomical structures. Perceived increased risk was infrequent, but some potential complications considered may be clinically important (e.g., nerve injury, local anaesthetic systemic toxicity). This pilot study provides evidence that such technology may support (rather than replace) clinician judgement for UGRA procedures.

\section{COMPARING THE EFFICACY OF PERICAPSULAR NERVE GROUP BLOCK (PENG) BLOCK VERSUS SUPRA-INGUINAL FASCIA ILIACA BLOCK (FIB) IN HIP ARTHROPLASTY}

C Vamshi*, DA Kumar, DC Sinha, DA Kumar, DS Kumar, DP Kumari. All India Institute of Medical Sciences, Patna, India

\subsection{6/rapm-2021-ESRA. 142}

Background and Aims Postoperative pain is a major concern in the patients undergoing Total Hip Arthroplasty. Peripheral nerve blockades have started gaining attention in the recent past for arthroplasty. Supra-inguinal fascia iliaca block (FIB) has become the standard of care at many places. Lately, (PENG) block has been proved to be safe and effective.

To compare the analgesic efficacy of USG guided Pericapsular nerve group block (PENG) with Supra-inguinal fascia iliaca block (SIFIB) in THR under General Anesthesia (GA).

Methods After IEC clearance, 18 patients scheduled to undergo THR under GA were included in this double blinded, randomized controlled trial.

Group I received PENG and Group II received SIFIB block with $0.25 \%$ bupivacaine and $1 \mathrm{mcg} / \mathrm{kg}$ clonidine respectively. The primary outcome was $24 \mathrm{hr}$ morphine consumption. Secondary outcomes were Intraoperative opioid consumption, Mean Numerical rating scale (NRS: 0-10) in $24 \mathrm{hr}$. and adverse effects.

Results Baseline demographics were similar. The mean 24-hour morphine(mg) consumption in Group I(PENG) was 10.44 $\pm 1.94 \mathrm{mg}$ and $12.22 \pm 1.85 \mathrm{mg}$ in Group II(SIFIB) with $\mathrm{p}$ value $=0.065$. Intraoperative Fentanyl consumption $(\mathrm{mcg})$ in PENG was $51.11 \pm 9.28$ and in SIFIB was $67.78 \pm 12.02$ with $\mathrm{p}$ value $=0.005$ which is statistically significant. Median of Mean NRS of $24 \mathrm{hr}$ was $2.63 \pm 0.44$ in PENG and 3.31 0.62 in SIFIB.
Conclusions Patients receiving PENG block required less intraoperative opioid. The postoperative 24-hour morphine consumption though not statistically significant but could be clinically significant. Also, PENG group experienced less pain comparing to SIFIB group. Two patients experienced vomiting in the SIFIB group.

IEC Clearance obtained with reference number: AIIMS/Pat/ IEC/PGTH/July19/22.

\section{PERIBULBAR BLOCK - A MATURE OPTION FOR AN IMMATURE PATIENT}

A Palha Ribeiro, LV Rodrigues*, P Pina. Centro Hospitalar Universitário do Porto, Porto, Portugal

\subsection{6/rapm-2021-ESRA. 143}

Background and Aims Literature is scarce regarding anesthetic management for treatment of retinopathy of prematurity (ROP). Retrospective analysis report that peribulbar block (PB) combined with general anesthesia (GA) seems safe in preterm infants.

Methods We report a case of a preterm infant submitted to bilateral PB for surgical treatment of ROP.

Results $2.8 \mathrm{~kg}, 41$ weeks correct age male preterm infant was scheduled for elective vitreoretinal surgery due to stage IV ROP.

In preoperative assessment no supplement oxygen was needed and he presented anemia and gastroesophageal reflux. Monitorization included continuous electrocardiogram, two pulse oximeters, noninvasive arterial pressure, end tidal carbon dioxide, temperature and glycemia. GA and tracheal intubation were performed and bilateral $\mathrm{PB}$ was executed with single infratemporal injection $0.25 \mathrm{ml} / \mathrm{kg} /$ eye ropivacaine $0.375 \%$ with $30 \mathrm{G}$ needle $(0.7 \mathrm{~mL}$ local anesthetic/eye). Maximum allowed concentration was considered $(3 \mathrm{mg} / \mathrm{kg})$.

Intraoperative course lasted 290 minutes and was uneventful, without further opioid requirements. Dexamethasone and paracetamol were administered. Neuromuscular blockage was reversed and infant successfully extubated and transferred to Neonatal Intensive Care Unit for 24 hours, following local protocol. No complications were reported or rescue analgesics were required.

Conclusions Premature infants present a challenge as they are more prone to perioperative cardiorespiratory complications.

$\mathrm{PB}$ has demonstrated effectiveness in reducing incidence of oculocardiac reflex, surgical bleeding, postoperative apnea and pain. GA complemented with bilateral PB achieved stable hemodynamics without additional opioid throughout surgery, indicating an acceptable quality of analgesia. Moreover, risk of postoperative apnea was reduced, as baby presented $<60$ weeks correct age and anemia, two known risk factors in preterm infants.

\section{TOTAL KNEE ARTHROPLASTY: CHRONIC PAIN AND FUNCTIONAL EVALUATION AFTER ONE YEAR, IMPLICATION OF THE FEMORAL NERVE BLOCK}

${ }^{1}$ A Maggiore*, ${ }^{2} \mathrm{~J}-\mathrm{F}$ Fils, ${ }^{3} \mathrm{~F}$ Zuccarini, ${ }^{1} \mathrm{E}$ Guntz. 'ULB, Braine L'Alleud, Belgium; ${ }^{2}$ ULB, Nivelles, Belgium; ${ }^{3}$ ULB, Bruxelles, Belgium

10.1136/rapm-2021-ESRA. 144 